\title{
Panorama da doença trofoblástica gestacional numa capital do nordeste
}

\author{
Panorama of gestational trophoblastic disease in a capital of the northeast
}

Panorama de la enfermedad Trofoblástica Gestacional en una capital del noreste

Hillary Gabriela dos Santos Oliveira ${ }^{1}$, Amuzza Aylla Pereira dos Santos ${ }^{1 *}$, Tâmara Silva de Lucena $^{1}$, Maria Elisângela Torres de Lima Sanches ${ }^{1}$, Maraysa Jéssyca de Oliveira Vieira ${ }^{1}$, Larissa de Morais Teixeira ${ }^{1}$, Julio Cesar Silva Oliveira ${ }^{1}$, Rosa Patrícia Gomes Tenório Omena Rodrigues ${ }^{1}$.

\section{RESUMO}

Objetivo: Analisar o panorama da doença trofoblástica gestacional em uma capital do Nordeste brasileiro no período de 2013 e 2018. Métodos: trata-se de um estudo quantitativo, descritivo por pesquisa documental, realizado através da análise de prontuários. Resultados: foram encontrados 238 prontuários de mulheres com diagnóstico de gestação molar, com uma média de 40 casos diagnosticados por ano. A maioria das mulheres do presente estudo estava na faixa etária entre 20 e 24 anos (28\%), era solteira $(71,1 \%)$, de cor/raça parda $(89,8 \%)$, com escolaridade de ensino médio completo/incompleto $(42,3 \%)$, e apresentava tipagem sanguínea $\mathrm{O}(54 \%)$ e fator Rh positivo (92\%). Quanto à história obstétrica, a maioria era primigesta $(42,3 \%)$, apresentou quadro de mola completa $(44 \%)$ e o tratamento de escolha mais utilizado foi a aspiração manual intrauterina $(44,4 \%)$. Conclusão: evidenciou-se que a doença trofoblástica gestacional é uma patologia que ocorreu numa pequena crescente no período de 2013 a 2018, mas com evolução clínica positiva.

Palavras-chave: Doença trofoblástica gestacional, Mola hidatiforme, Saúde da mulher.

\begin{abstract}
Objective: To analyze the panorama of gestational trophoblastic disease in a capital city of Northeast Brazil in the period of 2013 and 2018. Methods: this is a quantitative, descriptive and documentary study, carried out through the analysis of medical records. Results: 238 medical records of women diagnosed with molar pregnancy were found, with an average of 40 cases per year. Most women were in the age group between 20 and 24 years $(28 \%)$, were single $(71.1 \%)$, brown color $(89.8 \%)$, with complete / incomplete high school education (42.3\%), and had blood type O (54\%) and positive Rh factor (92\%). As for the obstetric history, most were on the first pragnancy $(42.3 \%)$, had a complete mole $(44 \%)$ and the treatment of choice was manual intrauterine aspiration (44.4\%). Conclusion: it became evident that gestational trophoblastic disease is a pathology that occurred in a small increase in the period from 2013 to 2018 , but with a positive clinical evolution.
\end{abstract}

Keywords: Gestational trophoblastic disease, Hydatidiform mole, Women's health.

\section{RESUMEN}

Objetivo: analizar el panorama del enfermedad trofoblástica gestacional en una ciudad capital del noreste de Brasil en el período de 2013 y 2018. Métodos: este es un estudio cuantitativo, descriptivo y documental, realizado a través del análisis de registros médicos. Resultados: se encontraron 238 registros médicos de mujeres diagnosticadas con embarazo molar, con un promedio de 40 casos por año. La mayoría de las mujeres pertenecían al grupo de edad entre 20 y 24 años (28\%), solteras $(71.1 \%)$, marrones $(89.8 \%)$, con

1 Universidade Federal de Alagoas Brasileira (UFAL), Maceió - AL. *E-mail: amuzza.santos@gmail.com

SUBMETIDO EM: 6/2020

ACEITO EM: 6/2020

PUBLICADO EM: 9/2020 
educación secundaria completa / incompleta (42.3\%) y tenían sangre tipo O (54\%) y factor Rh positivo $(92 \%)$. En cuanto a la historia obstétrica, la mayoría eran primigravida $(42,3 \%)$, tenían un resorte completo del $44 \%$ y el tratamiento de elección era la aspiración intrauterina manual $(44,4 \%)$. Conclusión: se hizo evidente que la enfermedad trofoblástica gestacional es una patología que ocurrió en un pequeño aumento en el período de 2013 a 2018, pero con una evolución clínica positiva.

Palabras clave: Enfermedad trofoblástica gestacional, Mola hidatiforme, Salud de la mujer.

\section{INTRODUÇÃO}

A Doença Trofoblástica Gestacional (DTG) é um grupo de condições incomuns associado com gravidez anormal, englobando um espectro de tumores com uma ampla gama de comportamento biológico e potencial para metástases distantes. A DTG refere-se a doenças benignas e malignas do espectro e inclui a mola hidatiforme (completa e parcial), a mola invasora, o coriocarcinoma, o tumor trofoblástico do local da placenta (PSTT) e o tumor trofoblástico epitelióide (ETT), sendo a Mola Hidatiforme responsável pela maior parte das DTG (SHAABAN AM, et al., 2017; NING F, et al., 2019)

Essas alterações se caracterizam pela proliferação anormal dos diferentes tipos de epitélio trofoblástico (citotrofoblasto, sinciciotrofoblasto e trofoblasto intermediário). O processo etiológico permanece desconhecido, mas acredita-se que a origem da DTG seja por gametogênese e fertilização anormais, além da transformação maligna do tecido trofoblástico (NGAN HYS, et al., 2018; MENDONÇA JBR, et al., 2016).

Os fatores de risco desta patologia incluem os extremos de idade, etnia, base genética, aborto espontâneo e restrição de nutrientes. Mulheres de 21 a 35 anos apresentam menor risco do que mulheres com mais de 35 anos e menos de 21 anos. Mulheres com histórico de aborto espontâneo prévio apresentam risco duas a três vezes maior de gravidez molar em comparação com a população em geral. Mulheres com histórico de gravidez molar têm um risco de 10 a 20 vezes de repetir a gravidez molar, e cerca de $20 \%$ das pacientes desenvolverão a forma maligna após a eliminação da mola (NING F, et al., 2019).

As molas hidatiformes são consideradas placentas imaturas muito edematosas, elas são divididas em molares completas e parciais. Uma gravidez molar completa ocorre quando um óvulo vazio é fertilizado por um espermatozoide, resultando no desenvolvimento de apenas partes da placenta, onde normalmente possui um cariótipo de $46, \mathrm{XX}$ e é de origem completamente paterna.

Já a mola parcial ocorre quando dois espermatozóides fertilizam um único óvulo, resultando no desenvolvimento de algumas ou de todas as partes fetais, possuindo um cariótipo triploide de $69, \mathrm{XXX}, 69$, XXY ou 69, XYY; no entanto, um cariótipo diplóide também pode existir (BRUCE S, SOROSKY J, 2019).

No Brasil não há estatística confiável, mas acredita-se que haja um caso dessa patologia para 200-400 gestações normais, o que a faz com que seja pouco frequente, e por isso desconhecida da população, e até mesmo de muitos profissionais de saúde. No Brasil, centros de referência do Rio de Janeiro e de Goiânia mostraram incidência de 18 por 1.000 nascidos vivos e de 8 por 1.000 partos, respectivamente. No centro de doenças trofoblásticas de Botucatu/SP, a incidência foi de 6,6 por 1.000 nascidos vivos (SOARES MKP, et al., 2015; MATOS M, et al., 2015).

Destaca-se que a importância de se trabalhar com essa temática justifica-se pelo fato de que a notificação de ocorrência da gestação molar no Brasil é considerada pouco confiável e seus dados epidemiológicos ainda pouco conhecidos, fato esse encontrado também no estado de Alagoas. Além disso, o estudo mostra-se relevante ao passo que se pretende apresentar dados reais relativos à gestação molar no estado de Alagoas e o perfil das mulheres acometidas (BRASIL, 2016; BRAGA A, et al., 2014).

Nessa perspectiva, esse artigo tem por objetivo: analisar o panorama dos casos de gestação molar em uma capital do Nordeste brasileiro no período de 2013 a 2018. 


\section{MÉTODOS}

Trata-se de um estudo descritivo, retrospectivo e documental, de abordagem quantitativa. A pesquisa foi realizada com dados referentes aos casos de mola gestacional na unidade de referência em uma capital do nordeste brasileiro. Para a composição da amostra, foi realizado um levantamento das mulheres que foram acometidas por essa patologia, onde se buscou seus prontuários entre os anos de 2013 e 2018.

Foram incluídos todos os prontuários de mulheres diagnosticadas com gestação molar, e excluídos aqueles que não possuíam dados completos para o estudo. Para a coleta de dados foi elaborado um formulário semiestruturado com dados de identificação para caracterizar a amostra, e dados referentes ao perfil obstétrico das mulheres relatado nos prontuários.

Assim, as variáveis utilizadas no estudo foram: preenchimento do formulário de coleta de dados, idade, cor/raça, estado civil, escolaridade, paridade, idade gestacional, procedimento realizado, tipo de mola e sinais e sintomas.

A coleta deu-se entre os meses de janeiro e fevereiro de 2019. Os dados foram coletados após aprovação do projeto pelo Comitê de Ética e Pesquisa da Universidade Federal de Alagoas, sob o processo CAAE no 92124318.2.0000.5013 no dia 21 de dezembro de 2018.

Sobre o Termo de Consentimento Livre e Esclarecido, foi solicitada a sua dispensa através de uma carta ao Comitê de Ética em Pesquisa o qual considerou sua dispensação, visto que se trata de pesquisa documental, através da análise dos prontuários das pacientes acometidas pela patologia, na qual não há contato e identificação de participantes da pesquisa.

Para a organização, tabulação e análise dos dados estatísticos descritivos utilizou-se o programa Microsoft Excel 2010. A análise descritiva ocorreu a partir de frequência absoluta (n) e percentual (F\%) e os resultados foram apresentados de forma descritiva, por meio de tabelas.

\section{RESULTADOS}

No total foram analisados 238 prontuários que relatavam diagnóstico patológico de Doença Trofoblástica Gestacional. Os prontuários apresentaram uma média de 40 casos por ano entre os anos de 2013 e 2018, e o ano de 2017 foi o que houve o maior número de casos (50 casos).

No que se refere ao perfil das mulheres atendidas e acompanhadas com diagnóstico de DTG nesse período, a maior parte estava dentro da faixa etária de 20-24 anos, representando $25,6 \%$. Quanto ao estado civil, $71,1 \%$ apresentaram estado civil solteira.

No que se refere à escolaridade, $43,3 \%$ possuíam o nível médio ou estava cursando-o. Em relação à raça/cor, $89,8 \%$ declarou-se de cor parda. Sobre a tipagem sanguínea, 54\% apresentou tipagem 0 , enquanto $92 \%$ possuía o fator Rh positivo (Tabela 1 ). 
Tabela 1 - Perfil epidemiológico das mulheres diagnosticadas com DTG com atendimento no período de 2013 a 2018 ( $N=238)$.

\begin{tabular}{|c|c|c|}
\hline Variável & $(n)$ & $(\%)$ \\
\hline \multicolumn{3}{|l|}{ Faixa etária } \\
\hline $10-14$ & 10 & 4,6 \\
\hline $15-19$ & 59 & 27,1 \\
\hline $20-24$ & 61 & 28 \\
\hline $25-29$ & 32 & 14,7 \\
\hline $30-34$ & 24 & 11 \\
\hline $35-39$ & 17 & 7,8 \\
\hline $40-44$ & 9 & 4,1 \\
\hline$>45$ & 6 & 2,8 \\
\hline \multicolumn{3}{|l|}{ Estado civil } \\
\hline Solteira & 143 & 71,1 \\
\hline Casada & 55 & 27,4 \\
\hline Divorciada & 1 & 0,5 \\
\hline União estável & 2 & 1,0 \\
\hline \multicolumn{3}{|l|}{ Escolaridade } \\
\hline Analfabeto ou não declarado & 59 & 24,8 \\
\hline Fundamental 1 & 5 & 2,1 \\
\hline Fundamental 2 & 59 & 24,8 \\
\hline Médio & 103 & 43,3 \\
\hline Superior & 12 & 5,0 \\
\hline \multicolumn{3}{|l|}{ Cor } \\
\hline Amarela & 1 & 0,5 \\
\hline Branca & 9 & 4,4 \\
\hline Parda & 184 & 89,8 \\
\hline Preta & 11 & 5,4 \\
\hline \multicolumn{3}{|l|}{ Grupo ABO } \\
\hline A & 49 & 27,8 \\
\hline B & 20 & 11,4 \\
\hline O & 95 & 54 \\
\hline$A B$ & 12 & 6,8 \\
\hline \multicolumn{3}{|l|}{ Fator $\mathbf{R H}$} \\
\hline Positivo & 162 & 92 \\
\hline Negativo & 14 & 8 \\
\hline
\end{tabular}

Fonte: Oliveira HGS, et al., 2019.

Quanto ao perfil obstétrico das mulheres, a maioria era primigesta $(42,3 \%)$. Em relação à paridade, $46,4 \%$ das mulheres eram nulíparas. No que se refere ao quantitativo de abortos, $19 \%$ mulheres tiveram 
aborto ou mola anterior, onde $59,6 \%$ sofreram 1 aborto, enquanto $6,4 \%$ tiveram mola anterior a atual (Tabela 2).

No que diz respeito à idade gestacional que estas mulheres descobriram a DTG, 37,8\% não constava nos prontuários analisados essa informação, bem como a data da última menstruação (DUM), enquanto $20,6 \%$ descobriram entre 7 e 10 semanas de gestação.

Tabela 2 - Perfil obstétrico das mulheres diagnosticadas com DTG com atendimento no período de 2013 a $2018(\mathrm{~N}=238)$.

\begin{tabular}{|c|c|c|}
\hline & (n) & $(\%)$ \\
\hline \multicolumn{3}{|l|}{ Gestações } \\
\hline Primigesta & 91 & 42,3 \\
\hline Secundigesta & 57 & 26,5 \\
\hline Tercigesta & 35 & 16,3 \\
\hline Multigesta & 32 & 14,9 \\
\hline \multicolumn{3}{|l|}{ Paridade } \\
\hline Nulípara & 91 & 46,4 \\
\hline Primímara & 59 & 29,6 \\
\hline Paucípara & 38 & 19,4 \\
\hline Multípara & 9 & 4,6 \\
\hline \multicolumn{3}{|l|}{ Abortos } \\
\hline 1 Aborto & 28 & 59,6 \\
\hline 2 Abortos & 12 & 25,5 \\
\hline 3 Abortos & 2 & 4,3 \\
\hline$>4$ Abortos & 2 & 4,3 \\
\hline Mola anterior & 3 & 6,4 \\
\hline Total & 47 & 19 \\
\hline \multicolumn{3}{|c|}{ Idade gestacional } \\
\hline 1-6 Semanas & 3 & 1,3 \\
\hline 7-10 Semanas & 49 & 20,6 \\
\hline 11-14 Semanas & 48 & 20,2 \\
\hline 15-18 Semanas & 30 & 12,6 \\
\hline 19-23 Semanas & 12 & 5,0 \\
\hline 24-28 Semanas & 3 & 1,3 \\
\hline >29 Semanas & 3 & 1,3 \\
\hline Não constava & 90 & 37,8 \\
\hline
\end{tabular}

Fonte: Oliveira HGS, et al., 2019.

No que concerne aos sinais e sintomas indicados pelas mulheres durante a admissão, 43,7\% não referiu queixa sintomática, enquanto $31,1 \%$ referiu somente sangramento transvaginal (STV) (Gráfico 1). 
Gráfico 1 - Distribuição quanto os sinais e sintomas indicados pelas mulheres diagnosticadas com DTG com atendimento no período de 2013 a 2018 na admissão ( $N=238)$.

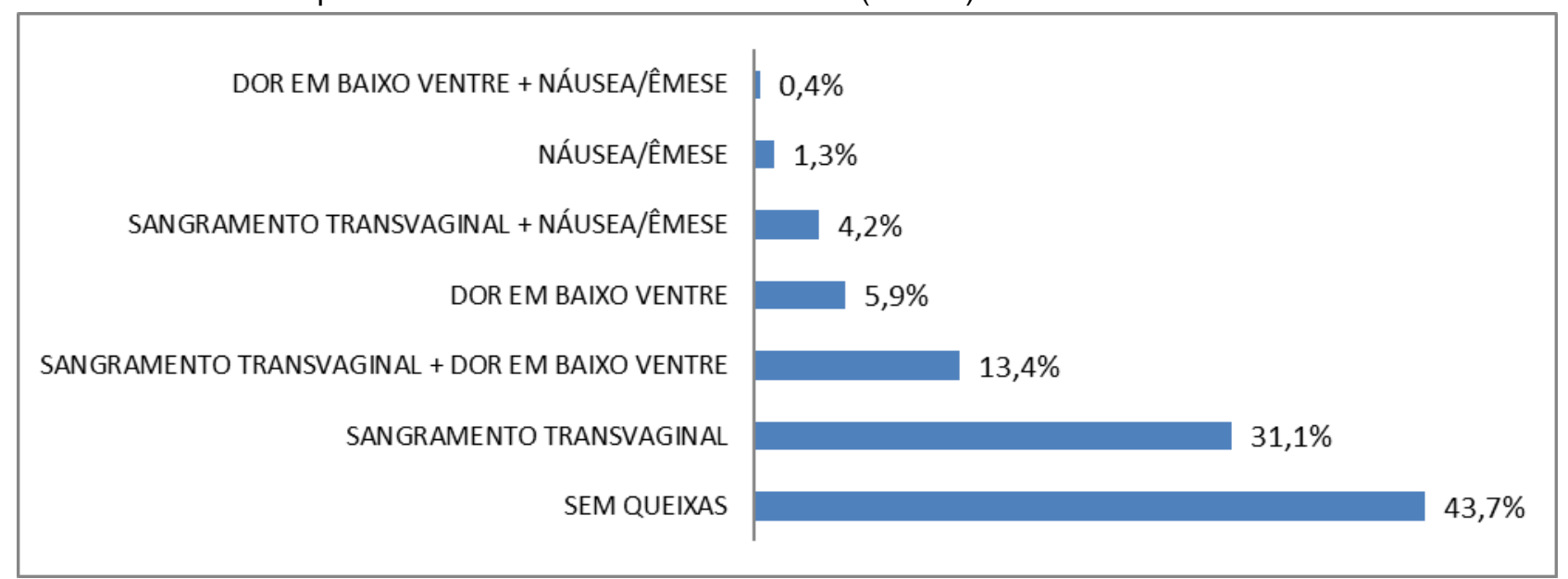

Fonte: Oliveira HGS, et al., 2019.

Sobre os tipos de DTG diagnosticados pela patologia clínica, o Gráfico 2 demonstra que $43,8 \%$ dos diagnósticos eram do tipo mola completa, num total de 82 pacientes, destacando que na maioria dos (156) prontuários não especificavam o tipo de mola encontrada.

Gráfico 2 - Distribuição quanto os tipos de DTG diagnosticados com atendimento no período de 2013 a $2018(\mathrm{~N}=82)$.

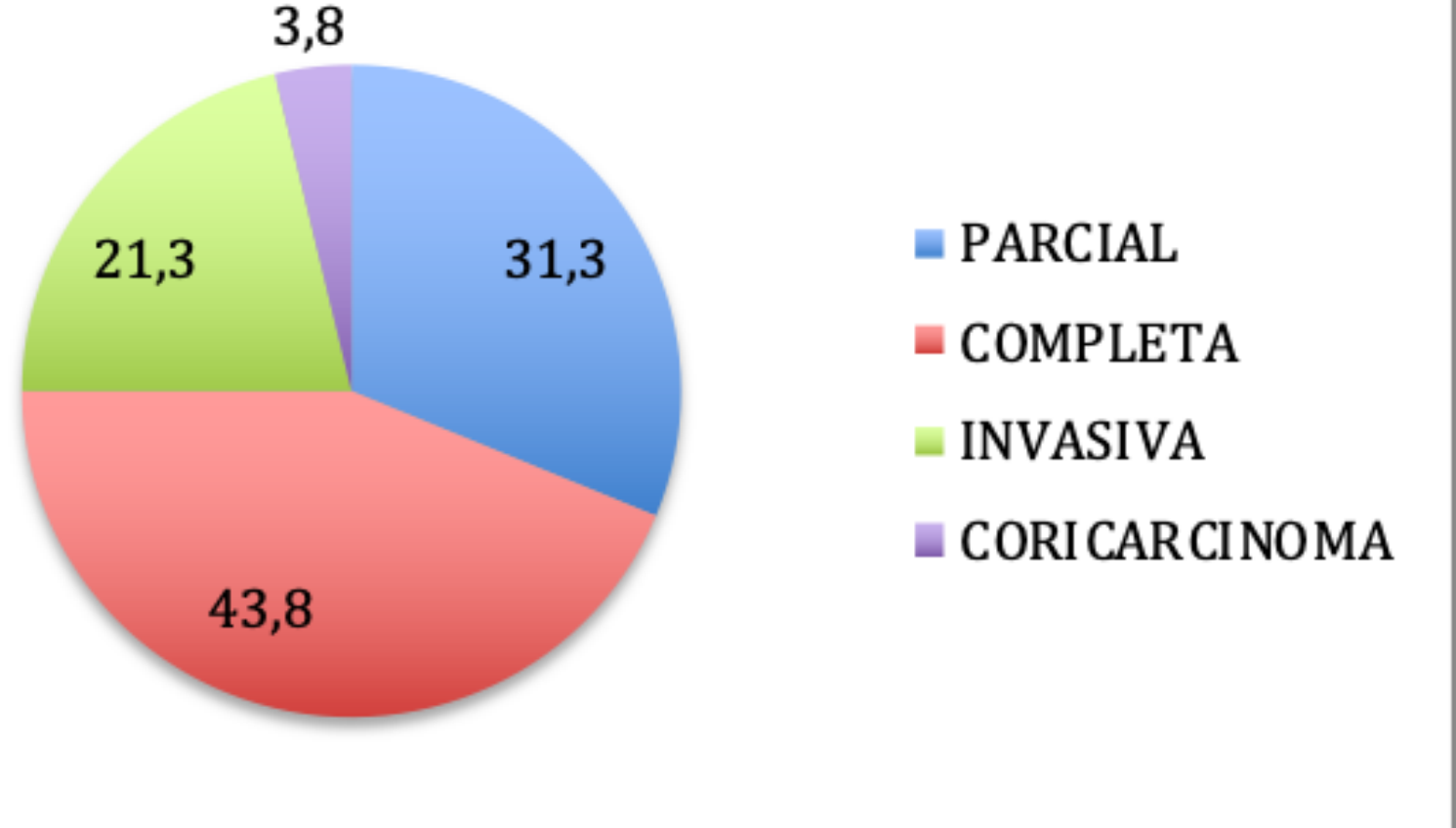

Fonte: Oliveira HGS, et al., 2019.

Relativo ao tipo de procedimento realizado após o diagnóstico ou suspeita de DTG, 44,4\% realizou a retirada do material pelo método de Aspiração Manual Intrauterina (AMIU). É possível ainda observar no mesmo gráfico, que houve casos em que a mulher necessitou realizar o mesmo ou outro procedimento mais de uma vez, devido a restos de material que permaneceram na cavidade uterina ou recidiva da patologia (Gráfico 3). 
Gráfico 3 - Distribuição das mulheres com diagnóstico de DTG quanto os tipos de procedimento realizado com atendimento no período de 2013 a 2018 ( $\mathrm{N}=238)$.

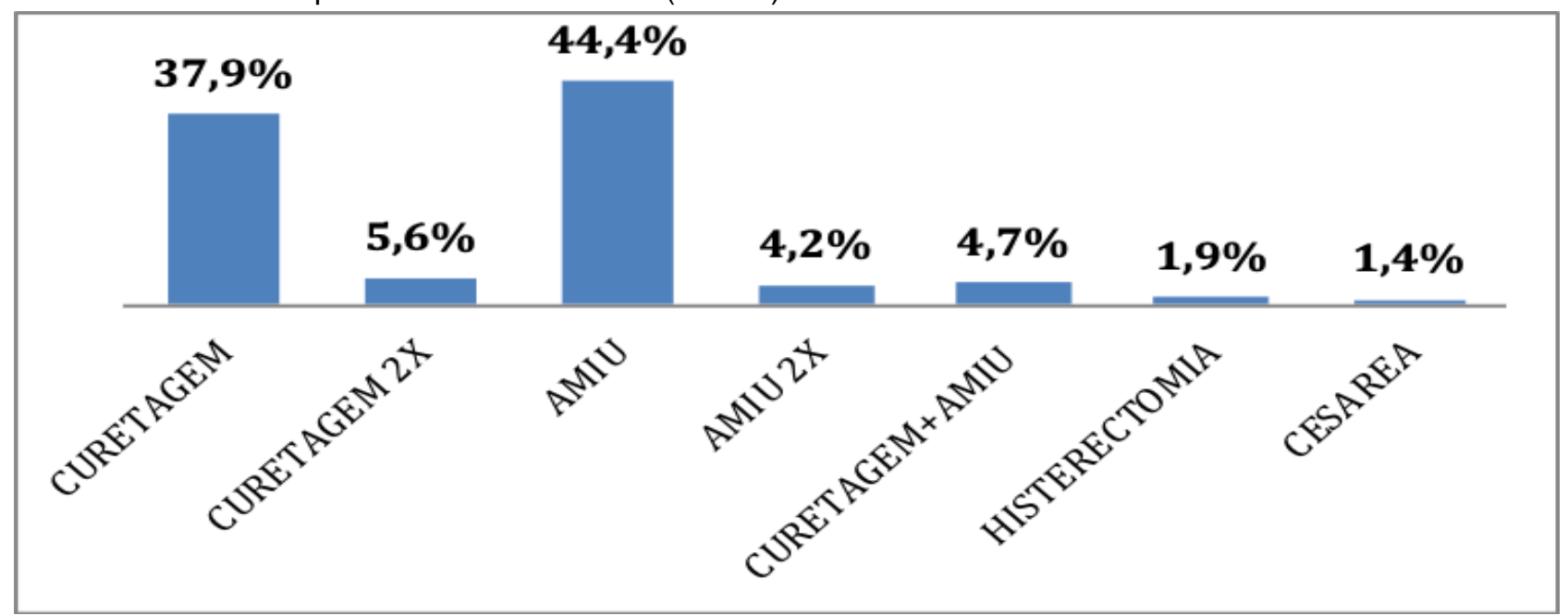

Fonte: Oliveira HGS, et al., 2019.

\section{DISCUSSÃO}

No estudo foi possível observar a importância de analisar o perfil de mulheres com gestação molar, visto ser uma patologia de origem pouco conhecida com diversos fatores de risco, como também associada aos extremos reprodutivos. De acordo com o Ministério da Saúde a idade fértil da mulher compreende-se entre 10 a 49 anos, sendo a idade materna um fator de risco para DTG, visto que a maior concentração da ocorrência acontece em mulheres com mais de 35 anos e menores de 18 anos de idade, sendo mais frequente em jovens (BRASIL, 2016).

No entanto, neste estudo observou-se que houve uma maior prevalência de casos de gestação molar entre as idades 20 a 24 anos, visto que corresponde a faixa etária onde a mulher encontra-se mais fértil. Em contrapartida, um estudo numa Maternidade Escola do Hospital das Clínicas da Universidade Federal de Goiás, mostrou que houve maior frequência em adolescentes entre 10 e 19 anos, faixa etária fora do apresentado nesse estudo (MENDONÇA JBR, et al., 2016).

Embora o risco seja alto para mulheres com idade avançada, devido à fertilização e/ou gametogênese anormal, a literatura ainda não traz justificativa da ocorrência desta patologia em mulheres jovens. Além disso, apesar de se constatar na literatura que os extremos de idade oferecem maior risco, a idade entre 2030 anos é onde as mulheres costumam engravidar mais, sendo uma possibilidade para o surgimento de tantos casos nessa faixa etária (FERRAZ L, et al., 2015).

Quanto à caracterização das mulheres, estas se apresentam como solteiras, cursando ou concluindo o ensino médio e de cor parda. Um estudo realizado na Colômbia que abordou a DTG, também mostrou que $44 \%$ das mulheres do estudo eram solteiras. Entretanto, apenas $16 \%$ das mulheres possuíam ou estavam cursando o ensino médio (VILLEGAS-MEJÍA CR, et al., 2017). Contudo, um nível de escolaridade maior pode auxiliar durante o processo patológico no que se refere ao seu entendimento, ainda que durante a rotina prática exista certa dificuldade para explanação da fisiopatologia, devido a sua complexidade e difícil compreensão.

Acerca da cor/raça referida, um estudo realizado em Florianópolis mostra que 90\% das mulheres eram de cor/raça branca, porém para classificação foi utilizado apenas as categorias raça branca e não branca. Uma revisão bibliográfica realizada no ano de 2018 diferentemente mostra que a patologia possui uma forte relação com a raça asiática (BISCARO A, et al., 2012; CORRÊA IB, et al., 2018). Essa diferença entre os estudos leva a questionar se realmente existe correlação entre a patologia e o aspecto cor/raça. Além disso, pode estar ligada à forma como os pesquisadores distinguem as diferentes raças, ou até estar relacionado ao local da pesquisa, como por exemplo, o Brasil que possui uma grande miscigenação. 
Já em relação à influência da tipagem sanguínea na DTG, este estudo mostra que a maioria das mulheres possuíam tipagem sanguínea do tipo $\mathrm{O}$ e fator Rh positivo, o que contrapõe ao descrito em um estudo no Hospital Universitário de Santander na Colômbia, o qual mostrou que a maioria das mulheres possuíam tipagem sanguínea A (VILLEGAS-MEJÍA CR, et al., 2017). Todavia, na literatura não possui nenhum descritivo sobre a correlação entre a tipagem sanguínea e a ocorrência da patologia. Além disso, no que concerne ao fator Rh, estudo realizado no Departamento de Patologia da Universidade Industrial de Santander entre os anos de 2005 e 2014, também revelou que o maior número de casos estava relacionado com o Rh positivo (RAMIREZ CAG, et al., 2018). Entretanto, estudo realizado em São Paulo com 214 pacientes que apresentaram diagnóstico de mola hidatiforme completa, atendidas consecutivamente no período de 1980 a dezembro de 2001, mostrou que a ausência do fator $\mathrm{Rh}$ foi associada a risco significante para o desenvolvimento de NTG (TIEZZI DG, et al., 2005).

É importante destacar que as pacientes Rh-negativo devem ser tratadas com imunoglobulina anti-D após o esvaziamento uterino, mesmo que as hemácias fetais não estejam presentes na mola completa, pois o trofoblasto expressa o fator $\mathrm{RhD}$, o que favorece a isoimunização. Esse é um fato importante para a assistência obstétrica, principalmente em relação à assistência prestada pela equipe de enfermagem, que deverá estar atenta a esse fator, a fim de que essa mulher possa receber o cuidado necessário durante sua internação, não havendo consequências futuras (CORRÊA IB, et al., 2018).

Outro fator importante para o desenvolvimento desta patologia é a história obstétrica dessas mulheres. De acordo com um estudo realizado em um Hospital no Cariri/CE, a DTG ocorre mais frequentemente em mulheres nulíparas, assim como foi observado neste estudo. Tal achado não corrobora com o estudo numa Maternidade em Goiás, onde a maioria era primípara. Além disso, outros fatores relacionados dizem respeito ao histórico pregresso de abortamento espontâneo e infertilidade. Neste estudo, quase $20 \%$ das mulheres sofreram algum aborto prévio, pouco mais do que o descrito no estudo do Cariri/CE, onde quase $10 \%$ sofreram algum tipo de aborto prévio. Além disso, apenas três das mulheres desse estudo tiveram caso de mola anterior, com resultado parecido com o estudo realizado em Goiás, onde apenas uma mulher apresentou histórico de DTG prévia (MENDONÇA BR, et al., 2016; SOARES MKP, et al., 2015).

De acordo com a Federação Internacional de Ginecologia e Obstetrícia, o antecedente pessoal de DTG aumenta o risco de gestação molar em gravidez subsequente. A importância de haver um diagnóstico correto ao ocorrer algum tipo de perda gestacional com suspeita de aborto é imprescindível, visto que a ocorrência de um aborto, principalmente de DTG prévia são fatores de grande risco para ocorrência em uma próxima gestação (SOARES MKP et al., 2015).

Em relação à idade gestacional em que ocorreu o diagnóstico de DTG, no geral, a mulheres não sabiam informar a data da última menstruação ou idade gestacional na qual se encontravam. Uma pequena parcela informou que se encontrava entre $7^{\mathrm{a}}$ a $10^{\mathrm{a}}$ semanas de gestação para todos os tipos de DTG, o que não se correlaciona com o estudo realizado na Colômbia, onde para mola completa o diagnóstico foi realizado entre 11,6 e 14,6 semanas de gestação e para mola parcial entre 11,4 e 17,1 semanas de gestação (RAMIREZ CAG et al., 2018).

Essa situação conta muito com o auxílio da atenção básica, visto que é imprescindível a captação da gestante antes de 12 semanas gestacionais, além da solicitação do exame de ultrassonografia no início do pré-natal, mesmo que o protocolo brasileiro Saúde das Mulheres (2016) fale que a decisão de incorporar ou não a ultrassonografia obstétrica à rotina do pré-natal deve considerar recursos disponíveis, qualidade dos serviços de saúde, bem como características e expectativas dos casais, e que o Ministério da Saúde através da Portaria no 650/2011, preconiza apenas uma ultrassonografia, porém o mesmo não refere à idade gestacional a ser realizada (BRASIL, 2016).

No tocante à sintomatologia das mulheres com gestação molar, a maioria delas não apresentou sintoma. Já as que apresentaram, muitas tiveram sangramento transvaginal. Devido ao uso de ultrassonografia e sua evolução tecnológica, as pacientes costumam ser diagnosticadas precocemente na gravidez e geralmente são assintomáticas nesse momento. Fato que muitas vezes pode ocorrer, pois a mulher sente-se ansiosa e prefere realizar o exame por contra própria e termina recebendo o diagnóstico da patologia sem ao menos apresentar os sintomas previstos (BRASIL, 2016; GONZÁLEZ RD, MORENA PB, 2018). 
Ainda sobre os sintomas que ocorreram, a literatura descreve que os mais comuns são sangramento vaginal, geralmente entre as semanas 6 a 16 semanas de gestação, dor em baixo ventre e hiperêmese. $O$ que mostra que esta patologia possui uma forte característica sintomatológica que pode auxiliar durante 0 processo de diagnóstico (SOARES MKP, et al., 2015; BOTELHO NM, et al., 2012).

No que concerne à diferenciação dos tipos de mola hidatiforme, nos casos em que foi realizado a diferenciação, a maioria das mulheres do presente estudo apresentou diagnóstico de mola completa, o que corrobora com o estudo realizado em Goiás onde o tipo clínico mais comum de DTG foi mola completa, assim como em um estudo na Colômbia. Entretanto, em muitos estudos na literatura, assim como neste, foi possível identificar a presença de diagnósticos de mola hidatiforme sem a classificação de seu tipo (MENDONÇA JBR, et al., 2016; RAMÍREZ CAG, et al., 2018). A ausência dessa classificação pode dificultar o percurso durante o período de seguimento pós-molar, pois um diagnóstico diferencial poderia auxiliar os profissionais de saúde que dão seguimento à assistência para essas mulheres sobre a possibilidade de uma evolução para uma NTG.

A neoplasia trofoblástica gestacional pode ocorrer após uma gravidez molar ou não molar. No estudo realizado no Pará, 22\% das mulheres desenvolveram NTG, o que corrobora com este presente estudo. Entretanto, uma coorte prospectiva realizada no período de 2001 a 2014 na Colômbia mostrou que 52\% de todos os casos pertenceram à mola invasora e $24 \%$ ao coriocarcinoma, sendo um número de grande discrepância aos dados mostrados neste estudo (VILLEGAS-MEJÍA CR, et al., 2017; SOARES MKP, et al., 2015). Nessa perspectiva, se faz necessário um diagnóstico fiel e precoce das NTG, visto que são patologias de tratamento diferenciado, havendo possibilidade de evolução para metástases, e em alguns casos até em óbito.

Quanto ao tratamento de escolha para DT, este é constituído por esvaziamento uterino através da técnica de vácuo-aspiração. A curetagem, como forma isolada de tratamento, não é recomendada devido à existência de alto risco de perfuração uterina no início do procedimento. A aspiração manual intrauterina (AMIU) é o método mais difundido no Brasil, de grande disponibilidade nas maternidades, podendo ser empregado em pacientes que desejam manter sua fertilidade, independente de tamanho uterino.

Além disso, mulheres que passam por esse procedimento apresentam recuperação mais rápida e alta com menos tempo de internação. Em mulheres com 40 anos ou mais e número de filhos definido, a histerectomia pode ser uma alternativa viável, posto que reduz a ocorrência de NTG pós-molar. Neste estudo, a maioria das mulheres realizou o esvaziamento com o método AMIU, seguido de curetagem convencional, o que concorda com o estudo realizado em Niterói, onde os procedimentos mais utilizados nesse contexto foram a vácuo aspiração e curetagem (FERRAZ L, et al., 2015; SOUSA MR, 2013).

Já a realização de um segundo procedimento é controversa. Pode ser benéfica para algumas pacientes que apresentam doença persistente, principalmente naquelas sem evidência histopatológica de doença invasora e com níveis de $\mathrm{HCG}<1.500 \mathrm{UI} / \mathrm{L}$, reduzindo a necessidade de quimioterapia. A necessidade de realização de um segundo procedimento cirúrgico, foi observado em 31 prontuários no presente estudo, o que contrapõe o estudo realizado em Niterói, onde somente uma mulher necessitou da realização de um segundo procedimento, assim como também houve a realização de uma histerectomia como neste estudo, o que mostra ser outra opção de tratamento, porém somente poderá ser indicada em pacientes que não desejam mais engravidar, e em pacientes acima de 40 anos (SOUSA MR, 2013; HAHN L, et al., 2018).

Dessa forma, destaca-se que o objetivo da pesquisa foi alcançado ao passo que foi analisado o panorama dos casos de gestação molar em uma capital do Nordeste brasileiro no período de 2013 a 2018, contribuindo com dados atualizados, através dos quais, os profissionais de saúde terão conhecimento mais acurado sobre a patologia e assim poderão exercer um cuidado íntegro, holístico e precoce às mulheres acometidas, promovendo consequentemente um sistema de notificação atualizado para melhor gerir e proporcionar uma rede de referência eficaz para o diagnóstico e tratamento da doença trofoblástica gestacional. 


\section{CONCLUSÃO}

A doença trofoblástica gestacional ocorreu numa pequena crescente entre as mulheres no período de 2013 a 2018. em uma capital do nordeste brasileiro. Quando foram analisadas as características das mulheres, observou-se que as mais acometidas por esta patologia são solteiras, de cor/raça parda, primigestas, cursando ou com ensino médio completo, possuem tipagem sanguínea $O$ positivo, e algumas apresentando sangramento transvaginal no momento do diagnóstico, porém a maioria delas não relatou sintoma. Um fato importante que deixou de ser relatado na maioria dos prontuários foi a idade gestacional da mulher no momento do diagnóstico. O tipo de doença trofoblástica gestacional mais relatado nos prontuários foi à mola completa, entretanto um grande número de mulheres não obteve o diagnóstico patológico de acordo com a tipologia, sendo uma possível falha do sistema quanto à análise patológica, o que pode prejudicar o desfecho clínico posteriormente, devido aos riscos de evolução para alguma neoplasia maligna e metástases. Com relação ao tratamento, a Aspiração Manual Intrauterina foi a escolhida pelos profissionais, pois na sua maioria é o tratamento de escolha para essa patologia, devido a diminuindo dos possíveis riscos que os outros tratamentos podem trazer. Contudo, a maioria dos casos evoluiu para um desfecho clínico positivo.

\section{REFERÊNCIAS}

1. BISCARO A, et al. Frequência de mola hidatiforme em tecidos obtidos por curetagem uterina. Rev. Bras. Ginecol. Obstet. 2012;34(6):254-258.

2. BOTELHO NM, et al. Aspectos clínicos da doença trofoblástica gestacional. Rev. Para. Med. 2012; 26(3).

3. BRAGA A, et al. Doença trofoblástica gestacional - atualização. Revista HUPE. 2014; 13(3):54-60.

4. BRASIL. Ministério da Saúde. Instituto Sírio Libanês de Ensino e Pesquisa. Protocolos da Atenção Básica - Saúde das mulheres. Brasília; 2016.

5. BRUCE S, SOROSKY J. Gestational Trophoblastic Disease. StatPearls, 2020.

6. CORRÊA IB, et al. Comorbidades associadas a mola hidatiforme: como diagnosticar e tratar. Revista de Patologia do Tocantins. 2018;5(3):68-74.

7. FERRAZ L, et al. Atualização no diagnóstico e tratamento da gravidez molar. JBM- 2015;103(2):6-12.

8. GONZÁLEZ RD, MORENA PB. Enfermedad Trofoblastica Gestacional. Med. leg. Costa Rica. 2018;35(1):30-43 HAHN L, et al. Doença trofoblástica gestacional: diagnóstico e tratamento. Oncologia: da prevenção ao tratamento. vol 39, n.2. Brasil: Acta medica; 2018; p. 136-150.

9. MATOS M, et al. Neoplasia trofoblástica gestacional após normalização espontânea da gonadotrofina coriônica humana em paciente com mola hidatiforme parcial. Rev Bras Ginecol Obstet. 2015;37(7):339-43.

10. MENDONÇA JBR, et al. Adesão ao seguimento ambulatorial de pacientes com doença trofoblástica gestacional atendidas em um centro de referência. REPROD CLIM. 2016;31(2):82-85.

11. MORAES FRR, RIBEIRO TA. Uso da radioterapia na neoplasia trofoblástica gestacional. Revista de Patologia do Tocantins. 2018; 5(4):47-50.

12. NGAN HYS, et al. Update on the diagnosis and management of gestational trophoblastic disease. Int $J$ Gynecol Obstet. 2018;143(2):79-85.

13. NING F, et al. Understanding and management of gestational trophoblastic disease. F1000 Research. 2019; 8:428

14. RAMÍREZ CAG, et al. Factores de riesgo, diagnóstico histológico y concentraciones de beta-hCG en pacientes con mola hidatiforme. MED.UIS. 2018; 31(1):39-46.

15. SHAABAN AM, et al. Gestational Trophoblastic Disease: Clinical and Imaging Features. Radiographics. 2017;37(2):681-700.

16. SOARES MKP, et al. Incidência de doença trofoblástica gestacional nos anos de 2007 a 2011 em um hospital público da região do Cariri. Cadernos ESP. 2015;9(1):35-41.

17. SOUSA MR. Características da doença trofoblástica gestacional em mulheres atendidas em uma maternidade no município de Niterói. Niterói: Universidade Federal Fluminense; 2013.

18. TIEZZI DG, et al. Fatores de risco para doença trofoblástica gestacional persistente. Rev. Bras. Ginecol. Obstet. $2005 ; 27(6): 331-9$

19. VILLEGAS-MEJÍA CR, et al. Enfermedad trofoblástica gestacional en un centro oncológico de Caldas - Colombia durante el periodo 2001 - 2014. Revista Médicas UIS. 2017;30(3):39-49. 